\title{
Clinician Encouragement and Online Health Record Usage
}

\author{
Bradley H. Crotty, MD, MPH', ${ }^{1,3}$, Aaron N. Winn, MPP, PhD 2,4, Onur Asan, PhD ${ }^{2,3,5}$, \\ Sneha Nagavally, $\mathrm{MPH}^{2}$, Rebekah J. Walker, $P \mathrm{PD}^{2,3}$, and Leonard E. Egede, MD, MS ${ }^{2,3}$
}

Check for

updates

${ }^{1}$ Collaborative for Healthcare Delivery Science, Medical College of Wisconsin, Milwaukee, WI, USA; ${ }^{2}$ Center for Advancing Population Science, Medical College of Wisconsin, Milwaukee, WI, USA; ${ }^{3}$ Division of General Medicine, Department of Medicine, Medical College of Wisconsin, Milwaukee, WI, USA; ${ }^{4}$ School of Pharmacy, Medical College of Wisconsin, Milwaukee, WI, USA; ${ }^{5}$ School of Systems and Enterprises, Stevens Institute of Technology, Hoboken, NJ, USA.

J Gen Intern Med 34(11):2345-7

DOI: $10.1007 / \mathrm{s} 11606-019-05162-9$

(c) Society of General Internal Medicine 2019

\section{INTRODUCTION}

Despite widespread adoption of electronic health records, under $30 \%$ of patients access their health records through portals. ${ }^{1}$ Patients who are at socioeconomic disadvantage, or of minority racial groups, have even lower adoption. ${ }^{2,3}$ Digitally engaging patients have several benefits for patient education, engagement, and practice efficiency. ${ }^{4}$ These effects are even more helpful among underserved patients. ${ }^{5}$ Access and usage of portals have also helped reduce quality gaps, such as vaccinations and screenings. ${ }^{6}$ To further realize gains in the digitization of medical data, having patients electronically interact with data and clinicians is critical to reaching a tipping point.

Prior research has found that clinician encouragement can change a variety of health behaviors. ${ }^{7}$ Therefore, we sought to assess the effect encouragement has on the access of patients' online health records. While demographics, internet connectivity, and illness burden have been previously explored and associated with adoption, this simple intervention of an active recommendation has not been fully explored.

\section{METHODS}

\section{Design, Setting, and Participants}

We used data from the Health Information National Trends Survey (HINTS5 Cycle 1), a nationally representative telephone-based survey of US households. Information regarding the sampling design and survey procedures is available at http://hints.cancer.gov. We restricted our analyses to respondents who were internet users, who had an outpatient visit within the previous 12 months, and who had complete data. We applied weights to develop population-based estimates for the pooled samples. Our primary outcome variable of interest was accessing one's online medical record within the last year. Among individuals that did access their record, we also examined use of secure messaging, test results,

Published online July 16, 2019 and prescription refills. Reporting encouragement, by clinicians or staff, was the primary dependent variable.

\section{Statistical Analysis}

To understand the relationship between clinician or staff encouragement and participant's likelihood we used a logistic regression with jackknife replication weights as appropriate for this survey design. We controlled for the following covariates: having a regular clinician, internet use, health insurance status, self-reported number of chronic conditions, and demographic factors including age, gender, race, and education.

\section{RESULTS}

We find close to $40 \%$ of patients access their online health record, with much higher rates if a patient received encouragement from their clinician $(15.0 \%$ vs $64.8 \%, P$ value $<$ $0.01)$. Individuals are more likely to receive encouragement if they are better educated, have higher income, have more chronic conditions, and are female (Table 1). Among individuals that accessed their online health record, physician or staff encouragement increased the probability of all aspects of portal usage. In our adjusted models for accessing their online health records, our results are consistent with the unadjusted results (Table 2). The odds of accessing an online record increases if a patient received encouragement from their clinician $(\mathrm{OR}=10.44,95 \% \mathrm{CI}=$ $7.26,15.00 ; P$ value $<0.01)$. The only other significant factor we found was having a regular clinician (OR = $2.32,95 \% \mathrm{CI}=1.48,3.65 ; P$ value $<0.01)$. Race, internet access, sex, and household income were not significantly associated with accessing the online health record after adjustment. Messaging, refilling a prescription, and reviewing test results online are all associated with receiving clinician encouragement (Table 2).

\section{DISCUSSION}

Encouragement from clinicians or their office staff has the strongest association with patients accessing their 
Table 1 Patient Characteristics

\begin{tabular}{|c|c|c|c|c|}
\hline & Overall & No encouragement & Encouragement & $P$ value \\
\hline$N$ & 1897 & 882 & 1015 & \\
\hline \multicolumn{5}{|l|}{ Age } \\
\hline $18-50$ & $58.7 \%$ & $57.3 \%$ & $58.0 \%$ & \multirow[t]{4}{*}{0.68} \\
\hline $51-65$ & $30.2 \%$ & $29.7 \%$ & $30.0 \%$ & \\
\hline $66-75$ & $8.0 \%$ & $9.1 \%$ & $8.6 \%$ & \\
\hline $76+$ & $3.1 \%$ & $3.9 \%$ & $3.5 \%$ & \\
\hline Female & $53.7 \%$ & $47.4 \%$ & $60.4 \%$ & $<0.01$ \\
\hline \multicolumn{5}{|l|}{ Race/ethnicity } \\
\hline White & $66.4 \%$ & $63.8 \%$ & $69.1 \%$ & \multirow[t]{5}{*}{0.38} \\
\hline Black & $9.0 \%$ & $9.1 \%$ & $9.0 \%$ & \\
\hline Hispanic & $13.9 \%$ & $16.3 \%$ & $11.4 \%$ & \\
\hline Asian & $4.7 \%$ & $4.7 \%$ & $4.7 \%$ & \\
\hline Other/missing & $6.0 \%$ & $6.2 \%$ & $5.8 \%$ & \\
\hline \multicolumn{5}{|l|}{ Type of internet use } \\
\hline Dial-up & $2.6 \%$ & $2.6 \%$ & $2.5 \%$ & \multirow[t]{4}{*}{0.28} \\
\hline Broadband & $56.0 \%$ & $59.3 \%$ & $52.6 \%$ & \\
\hline Cell & $25.4 \%$ & $24.1 \%$ & $26.8 \%$ & \\
\hline Wi-Fi & $16.0 \%$ & $14.0 \%$ & $18.1 \%$ & \\
\hline \multicolumn{5}{|l|}{ Education } \\
\hline High school or less & $23.2 \%$ & $28.3 \%$ & $17.9 \%$ & \multirow[t]{3}{*}{$<0.01$} \\
\hline Some college & $33.5 \%$ & $33.3 \%$ & $33.7 \%$ & \\
\hline College & $43.3 \%$ & $38.4 \%$ & $48.4 \%$ & \\
\hline \multicolumn{5}{|l|}{ Household income } \\
\hline Less than $\$ 20,000$ & $11.0 \%$ & $13.7 \%$ & $8.2 \%$ & \multirow[t]{5}{*}{$<0.01$} \\
\hline$\$ 20,000-34,999$ & $10.7 \%$ & $12.9 \%$ & $8.4 \%$ & \\
\hline$\$ 35,000-49,999$ & $12.7 \%$ & $13.8 \%$ & $11.5 \%$ & \\
\hline$\$ 50,000-74,999$ & $20.3 \%$ & $20.4 \%$ & $20.2 \%$ & \\
\hline$\$ 75,000+$ & $45.3 \%$ & $39.2 \%$ & $51.8 \%$ & \\
\hline Has a chronic condition & $63.8 \%$ & $58.5 \%$ & $69.4 \%$ & $<0.01$ \\
\hline Has a regular clinician & $73.9 \%$ & $69.9 .5 \%$ & $78.2 \%$ & $<0.01$ \\
\hline \multicolumn{5}{|l|}{ Online portal utilization } \\
\hline Access online record & $39.3 \%$ & $15.0 \%$ & $64.8 \%$ & $<0.01$ \\
\hline Message, among access online users & $48.3 \%$ & $34.2 \%$ & $51.9 \%$ & 0.01 \\
\hline Refill prescription online, among access online users & $37.0 \%$ & $25.9 \%$ & $39.8 \%$ & 0.02 \\
\hline Reviewing test results online, among access online users & $84.6 \%$ & $72.0 \%$ & $87.7 \%$ & $<0.01$ \\
\hline
\end{tabular}

health record online. We also found that encouragement was strongly associated with use of portal functions. Encouragement may mediate enrollment and usage in several potential ways: raising awareness, providing acceptance of use, and transferring a sense of usefulness that patients may not identify on their own.

Given that only clinicians offering patient portals will encourage patients to use them, the result should be interpreted with some caution. However, since $95 \%$ of acute care hospitals and $63 \%$ of office-based physicians offered portals in this time period, ${ }^{6}$ and the effect size is large, we believe the overall finding would remain statistically significant and large in magnitude. Moreover, our secondary outcomes only included individuals that accessed their records, which ensures that clinicians offered a patient portal, and we find consistent associations that encouragement is associated with use.

This study finds that clinician encouragement is a strongly associated with patients accessing their online health record. Making portal encouragement part of a standard office visit may help enroll patients in other digital services and may help catalyze the tipping point toward digital care.

Table 2 Adjusted Association Between Receiving Physician Encouragement and Accessing Online Electronic Medical Records

\begin{tabular}{lrr}
\hline \hline & Odds ratios & $P$ value \\
\hline Access online record & $10.44(7.26,15.00)$ & $<0.01$ \\
Message, among access online users & $1.98(1.16,3.39)$ & 0.01 \\
Refill prescription online, among access online users & $1.92(1.06,3.49)$ & 0.03 \\
Reviewing test results online, among access online users & $2.82(1.27,6.27)$ & 0.01 \\
\hline
\end{tabular}

Authors' analysis of the Health Information National Trends Survey (HINTS5) survey for individuals that use the internet and had an outpatient visit within the past year. Odds ratios generated from a logistic regression using jackknife replication weights adjusting for age, gender, race, insurance, type of internet used, education region, household income, number of chronic conditions, and having a regular clinician 
Corresponding Author: Bradley H. Crotty, MD, MPH; Collaborative for Healthcare Delivery ScienceMedical College of Wisconsin, Milwaukee, WI, USA (e-mail: bcrotty@mcw.edu).

Funding Information Effort for this study was partially supported by the National Institute of Diabetes and Digestive Kidney Disease (K24DK093699, PI: Egede).

\section{Compliance with Ethical Standards:}

Conflict of Interest: Dr. Crotty reports serving as an advisor to Buoy Health LLC. All remaining authors declare that they do not have a conflict of interest.

\section{REFERENCES}

1. Peacock S, Reddy A, Leveille SG, et al. Patient portals and personal health information online: perception, access, and use by US adults. J Am Med Inform Assoc. 2016;24(e1):e173-e177.
2. Yamin CK, Emani S, Williams DH, et al. The digital divide in adoption and use of a personal health record. Arch Intern Med. 2011;171(6):568574.

3. Graetz I, Gordon N, Fung V, Hamity C, Reed ME. The Digital Divide and Patient Portals: Internet Access Explained Differences in Patient Portal Use for Secure Messaging by Age, Race, and Income. Med Care. 2016;54(8):772-779.

4. Delbanco T, Walker J, Bell SK, et al. Inviting patients to read their doctors' notes: a quasi-experimental study and a look ahead. Ann Intern Med. 2012;157(7):461-470.

5. Gerard M, Chimowitz H, Fossa A, Bourgeois F, Fernandez L, Bell SK. The Importance of Visit Notes on Patient Portals for Engaging Less Educated or Nonwhite Patients: Survey Study. J Med Internet Res. 2018;20(5):e191.

6. Wright A, Poon EG, Wald J, et al. Randomized controlled trial of health maintenance reminders provided directly to patients through an electronic PHR. J Gen Intern Med. 2012;27(1):85-92.

7. Kreuter Mw, Chheda SG, Bull FC. How does physician advice influence patient behavior? Evidence for a priming effect. Arch Fam Med. 2000;9(5):426-433.

Publisher's Note Springer Nature remains neutral with regard to jurisdictional claims in published maps and institutional affiliations. 